GARCIA, C. A.; SANTOS, A. G.; BARREIRA, S. Estrutura fitossociológica de uma área de cerrado na estação ecológica de Itapeva, São Paulo, Brasil. Revista de Agricultura Neotropical, Cassilândia-MS, v. 2, n. 2, p. 77-85, abr./jun. 2015.

\title{
ESTRUTURA FITOSSOCIOLÓGICA DE UMA ÁREA DE CERRADO NA ESTAÇÃO ECOLÓGICA DE ITAPEVA, SÃO PAULO, BRASIL
}

\section{CELSO AUGUSTO GARCIA ${ }^{1}$, ARNALDO GONÇALVES DOS SANTOS ${ }^{1}$, SYBELLE BARREIRA $^{2}$}

\footnotetext{
${ }^{1}$ Geoflor Consultoria Florestal, Barueri/SP, celso.garcia@geoflor.com.br; ${ }^{2}$ Universidade Federal de Goiás, Escola de Agronomia, Goiânia/GO, Brasil, sbarreir@esalq.usp.br
}

\begin{abstract}
RESUMO: A Estação Ecológica de Itapeva, SP é uma unidade de conservação administrada pelo Instituto Florestal do Estado de São Paulo e está instalada em uma área de 106,77 hectares. Com o objetivo fornecer subsídios para futuros projetos de manejo do local, bem como conhecer a estrutura da vegetação, foram calculados alguns parâmetros fitossociológicos, utilizando-se a metodologia de pontos quadrantes. Foram amostrados 800 indivíduos, pertencentes a 18 famílias botânicas totalizando 30 espécies. A distância média geral de ponto-planta foi de $1,81 \mathrm{~m}$ fornecendo uma área média geral de $3,85 \mathrm{~m}^{2}$, sendo a densidade total por área de 2.870 árvores ha $^{-1}$. A família Fabaceae teve o maior número de espécies amostradas (cinco espécies) seguida por Lauraceae (três), Melastomataceae (duas), Araliaceae (duas) e Erythroxylaceae (duas). As espécies listadas são comuns no cerrado do estado de São Paulo, no entanto não são exclusivas, sendo ocorrentes também em formações paludículas e floresta ombrófila mista. Os resultados apontam para uma área perturbada, com a descaracterização do cerrado original e invasão de outras espécies, apontando para a necessidade urgente de um estudo mais aprofundado que permita desenvolver estratégias de recuperação e preservação da área.
\end{abstract}

PALAVRAS-CHAVE: levantamento florístico, unidade de conservação, bioma cerrado.

\section{PHYTOSOCIOLOGICAL STRUCTURE OF A SAVANNAH AREA IN THE ECOLOGICAL STATION ITAPEVA, SAO PAULO STATE, BRAZIL}

\begin{abstract}
The Itapeva (SP) Ecological Station is a conservation unit that is administered by the State of Sao Paulo Forestry Institute and has 106.77 hectares of land. In order to provide subsidies to future plans of the management of the place, as well as to know the vegetation structure, some phytosociological parameters were calculated using the quadrantpoint method 800 individuals were sampled; they belong to 18 bothanical families, in a total of 30 species. The general average distance of the point-plant was of $1.81 \mathrm{~m}$, providing a general average area of $3.85 \mathrm{~m}^{2}$ and the total density by area was of 2.870 trees $\mathrm{ha}^{-1}$. The Fabaceae family had the largest number of sampled species (five species), followed by the Lauraceae family (three), the Melastomataceae (two), the Araliaceae (two) and the Erythroxylaceae (two). The enumarated species are common in the SP Cerrado, notwithstanding they are not exclusive as they also occur in paludicola formations and mixed ombrophylous forest. The results point to a perturbed area, with the degradation of the native Cerrado and the invasion of other species, what suggests the urgent demand of a more accurate study that may allow the development of recovery and the preservation of the area.
\end{abstract}

KEY WORDS: floristic survey, protected area, cerrado. 


\section{INTRODUÇÃO}

A formação de Cerrado cobria originalmente cerca de $23 \%$ do território brasileiro, ou aproximadamente dois milhões de $\mathrm{km}^{2}$, abrangendo os Estados de Goiás, Tocantins, Mato Grosso, Mato Grosso do Sul, Bahia, Minas Gerais, Ceará, Maranhão, Piauí, Rondônia, São Paulo e Paraná, além de algumas áreas disjuntas do Amapá, Amazonas, Pará e Roraima (RIBEIRO; WALTER, 1998). Atualmente ocupa menos de 1\% do território paulista (DURIGAN et al, 2003).

Visando preservar remanescentes de cerrado no Estado de São Paulo foi criada pelo Decreto Estadual n $n^{\circ} .23 .791$, de 13 de agosto de 1985, a Estação Ecológica de Itapeva (EEI) com a finalidade de proteger amostras de ecossistemas de cerrado, campo-cerrado e campo, importantes para a reprodução de animais e nidificação de aves ameaçadas de extinção (SÃO PAULO, 1998).

Na EEI predomina o bioma Cerrado, com vegetação em estágio avançado de sucessão além de vegetação secundária e campos com forte ação humana. Embora seja uma importante área dentre as administradas pelo Instituto Florestal de SP, a EEI não possui plano de manejo e poucos estudos foram realizados na área, dentre os quais os de Cielo-Filho et al (2012).

Com o propósito de conhecer e caracterizar a vegetação da EEI a fim de colaborar para a manutenção dos processos ecológicos, bem como para o desenvolvimento de plano de manejo para a Unidade de Conservação (UC) este trabalho realizou as seguintes etapas: a) realizar levantamento florístico da vegetação; b) realizar uma análise fitossociológica; c) fornecer subsídios para o desenvolvimento de estratégias de conservação in situ de espécies valiosas.

\section{MATERIAL E MÉTODOS}

A Estação Ecológica de Itapeva (EEI) localizada à margem da rodovia Estadual

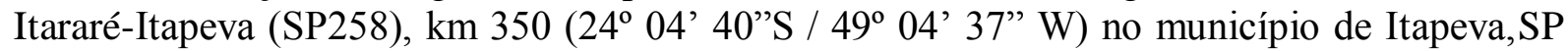
(Figura 1) possui 106,77 há. O clima da região é o do tipo cfb, segundo a classificação de Köppen, e a temperatura média anual é de $18,5^{\circ} \mathrm{c}$. Possui relevo levemente ondulado Latossolo Vermelho Amarelo e o Argissolo Amarelo, mas ocorrem também associações de Argissolo Amarelo+Neossolo Litólico e Gleissolo Melânico+Gleissolo Háplico (NOVAIS et al. 2009)

A amostragem da vegetação foi realizada através do método de Pontos Quadrantes, que segundo Krebs (1999) consiste no estabelecimento de inúmeros pontos na área de estudo, os quais atuam como centro de um plano cartesiano que define quatro quadrantes. É importante que a distância entre os pontos seja determinada de maneira a evitar que um mesmo indivíduo seja amostrado em dois pontos distintos.

Em cada quadrante é marcado e identificado o indivíduo mais próximo do ponto central que atenda aos critérios de inclusão da amostragem e, em seguida, é registrada a distância deste em relação ao ponto central do quadrante. São registradas também a altura e a circunferência ou o diâmetro da planta.

Para este trabalho foram selecionados 40 ha no interior da EEI, onde foram alocados 10 transectos e estabelecidos 200 pontos de amostragem. Estes pontos foram colocados em distância de 20 m, espaçados numa distância de 100 m (Figuras 2 e 3). 


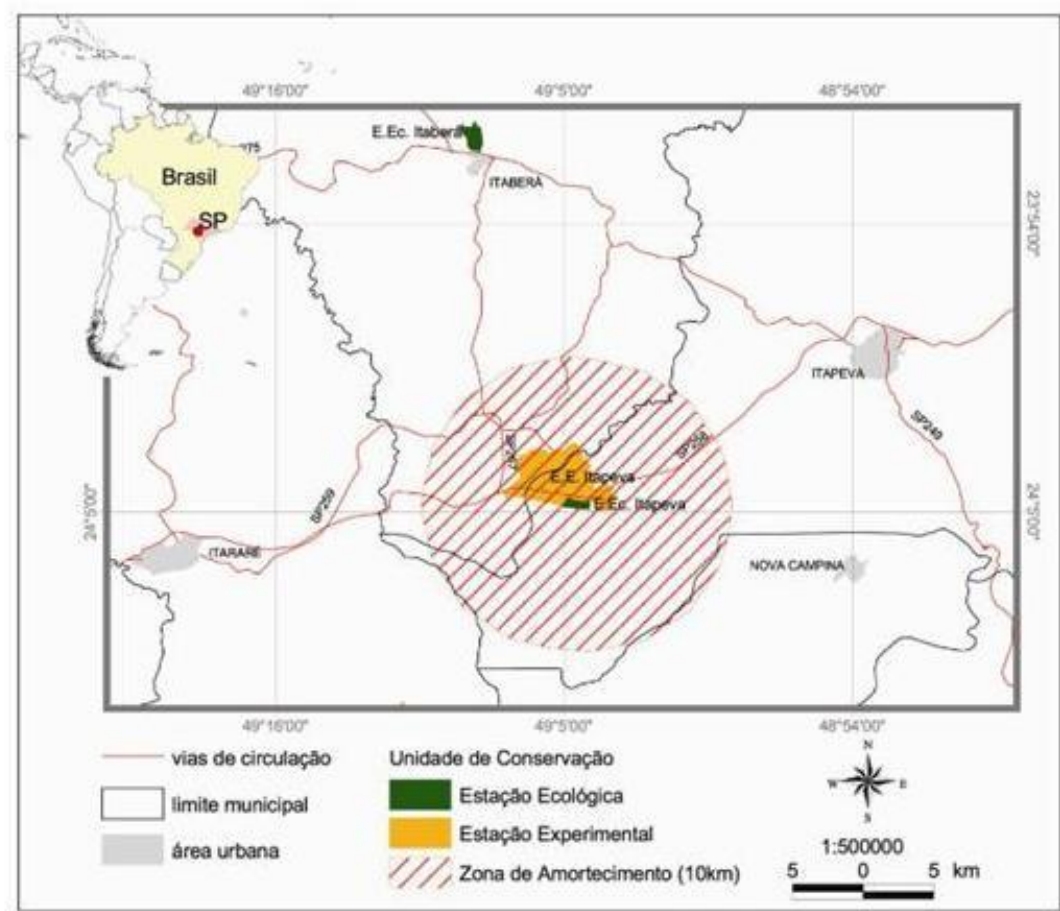

Figura 1. Mapa de localização da Estação Ecológica de Itapeva (EEI)

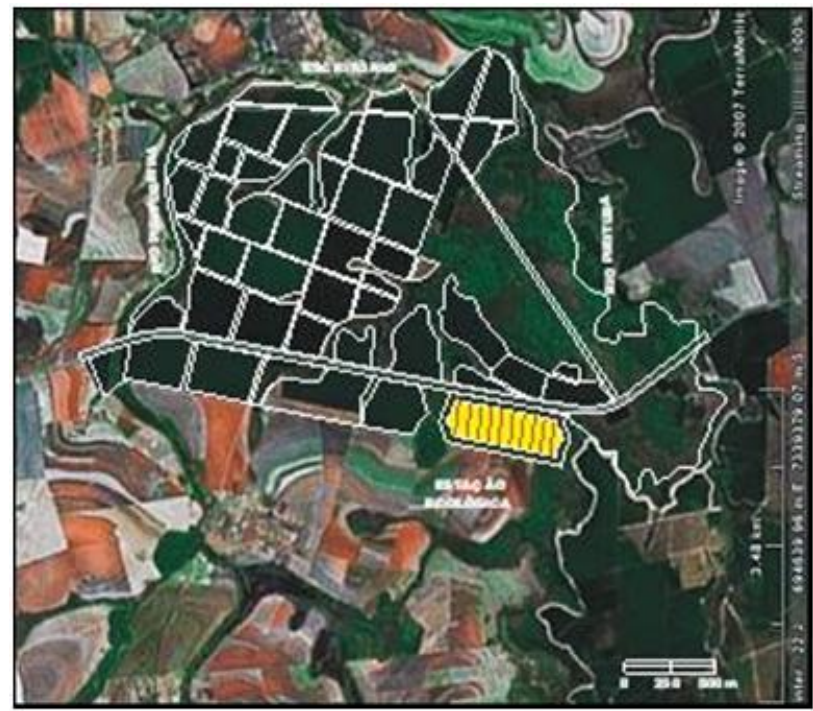

Figura 2. Imagem do Satélite LAND SAT 7/ETM, localização georeferenciada da grade amostral na área de estudo da E. Ec. de Itapeva

Os quadrantes foram estabelecidos em cada ponto de amostragem seguindo medições de ponto-planta, perímetro basal e área de cobertura de cada árvore amostrada. Todos os indivíduos marcados tiveram amostras botânicas coletadas para identificação em herbário com base em literatura especializada e comparação com o acervo, ainda assim, alguns indivíduos somente puderam ser identificados ao nível de gênero. A classificação das espécies seguiu o sistema do Angiosperm Phylogeny Group III (APG III, 2009) e a nomenclatura botânica com as abreviações dos respectivos autores foi conferida segundo o site "Flora Brasil” (LISTA DE ESPÉCIES DA FLORA DO BRASIL, 2014). 


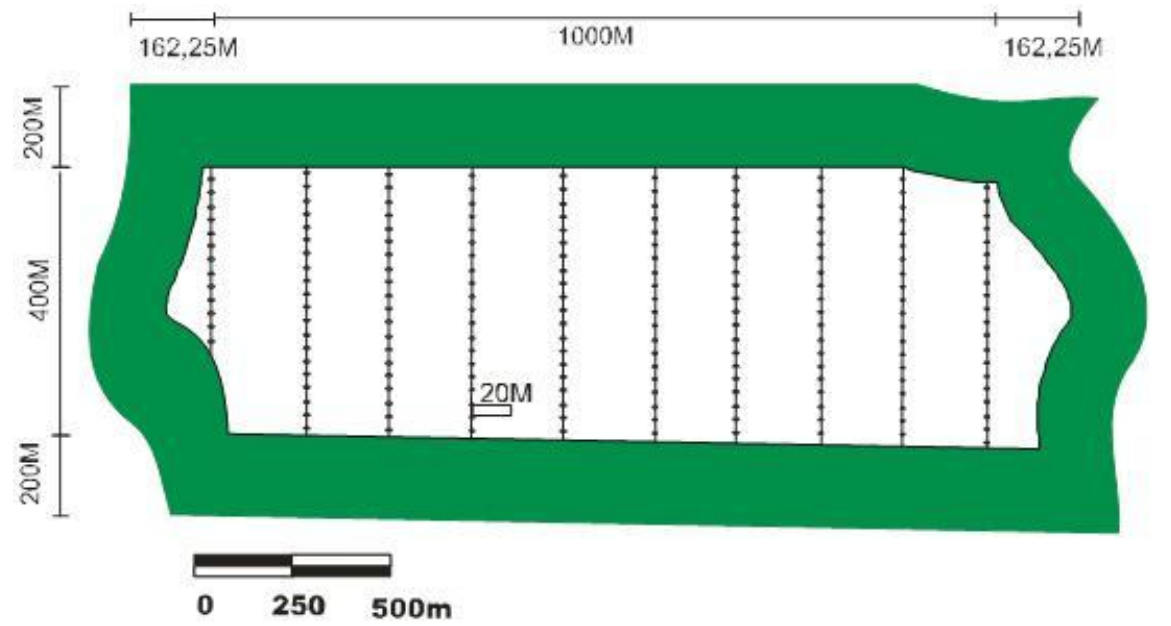

Figura 3. Esquema da amostragem na Estação Ecológica de Itapeva, SP

Foram amostrados na grade dos pontos quadrantes os indivíduos arbóreos com Perímetro do tronco à altura do Peito $(\mathrm{PAP})$ igual ou superior a $05 \mathrm{~cm}$. As árvores mortas em pé não foram incluídas na amostragem.

As estimativas de índices fitossiciológicos foram calculadas com base em MüllerDombois; Ellenberg (1974). A estrutura da comunidade vegetal foi calculada a partir dos parâmetros fitossociológicos: Densidade Relativa (DR), Dominância Relativa (DoR), Frequência Relativa (FR) e Índice de Valor de Importância (IVI) (MUELLER-DOMBOIS; ELLENBERG, 1974), bem como o índice de diversidade de Shannon-Wiener (H') (MAGURRAN, 1988). A média geométrica G foi calculada com base em Spiegel (1976).

\section{RESULTADOS E DISCUSSÃO}

Nos 200 pontos foram amostrados 800 indivíduos, com média geométrica de 2,42 indivíduos/ponto. A distância média geral de ponto-planta foi de $1,81 \mathrm{~m}$, fornecendo uma área média geral de $3,85 \mathrm{~m}^{2}$, sendo este o valor médio da área ocupada por uma árvore de médio porte qualquer, sendo a densidade total por área de 2.870 árvores/ha. Os indivíduos amostrados pertencem a 18 famílias botânicas, 23 gêneros e 30 espécies, sendo 03 não identificadas, nem mesmo ao nível de família (Tabela 1).

A família Fabaceae teve o maior número de espécies amostradas (cinco) seguida por Lauraceae (três), Melastomataceae (duas), Araliaceae (duas) e Erythroxylaceae (duas) e apenas um indivíduo de cada espécie representou 13 famílias. Estas cinco famílias colaboraram com $46,67 \%$ de toda a riqueza encontrada na área. Estudos realizados em Cerrados de Goiás e do Distrito Federal também revelaram a predominância da família Fabaceae (SAPORETTI Jr et al., 2003; ASSUNÇÃO; FELFILI, 2004; SOARES et al., 2012).

Espécies encontradas na área de estudo também são relatados nos trabalhos de Durigan et al (2002), Fidelis e Godoy (2003) e Paula et al (2015), Reys et al (2013). No entanto, algumas não são comumente encontradas nesta formação, tendo sido reportadas em formações paludícolas, como por exemplo, Clethra scabra Pers, (TORRES et al. 1994, TONIATO et al. 1998) ou então sendo típicas da floresta ombrófila mista no sul, como Ilex paraguariensis A.St.-Hil. (SANQUETTA et al, 2002). A invasão de espécies que não são típicas da vegetação, como o Pinus elliottii Engelm foi relata por Almeida et al (2010). 
Do ponto de vista do manejo da unidade, portanto, a invasão biológica dessa fisionomia é bastante preocupante, pois representa a possibilidade de perda de uma porção considerável da biodiversidade que a Estação visa proteger (ALMEIDA et al, 2010).

Os parâmetros fitossociológicos para cada espécie estão listados na Tabela 2; as espécies com maiores valores de IVI foram Scheflera macrocarpha (Cham. \& Schltdl.), Anadenanthera peregrina (L.) var. falcata (Benth) e Nectandra cuspidata Nees. Em um estudo realizado em área de cerrado em Santa Rita do Passa Quatro/SP, Fidelis e Godoy (2003) apontam Anadentanthera peregrina var. falcata como o maior valor de importância. No trabalho realizado por Reys et al (2013) a Anadenanthera peregrina (L.) apresentou maior IVI contrariando o que foi encontrado no presente estudo. Scheflera macrocarpha (Cham. \& Schltdl.) apresenta maior IVI pois apesar da baixa densidade observada apresenta elevada dominância.

Com relação à dominância, as espécies mais representativas de acordo com sua área basal são consideradas típicas das formações de cerrado como: Syagrus romanzoffiana (cham.) glassm, Stryphnodendron pulcherrimum (Willd.) Hochr., Anadenanthera peregrina (L.) Speg. var. falcata (Benth), Anadenanthera peregrina (L.), Gochnatia polymorpha Less. e Schefflera macrocarpa (Cham. \& Schltdl.).

Quanto à freqüência, as espécies que mais se destacaram foram: Rapanea sp, Copaifera langsdorffii Desf., Cupania vernalis Cambess., Nectandra cuspidata Nees, Anadenanthera peregrina (L.) Speg. var. falcata (Benth) e Schefflera vinosa (Cham. \& Schltdl.).

Tabela 1. Lista de espécies identificadas na área de estudo

\begin{tabular}{|c|c|c|}
\hline Família & Espécie & Nome popular \\
\hline Anonnaceae & Xylopia sericea St. Hill. & Pindaíba \\
\hline Apocynaceae & Aspidosperma tomentosum Mart. & Guatambú \\
\hline Aquifoliaceae & Ilex paraguaiensis A.St.-Hil. & Erva mate \\
\hline Araliaceae & Scheflera macrocarpa (Cham. \& Schltdl.) & Mandiocão \\
\hline Araliaceae & Scheflera vinosa (Cham. \& Schltdl.) & Mandioquinha \\
\hline Arecaceae & Syagrus romanzoffiana (cham.) glassm & Jerivá \\
\hline Asteraceae & Gochnatia polymorpha Less. & Cambará \\
\hline Bignoniaceae & Handroanthus chrysotrichus (Mart. ex DC.) Mattos & Ipê amarelo \\
\hline Burceraceae & Protium heptaphyllum March. & Almecegueira \\
\hline Clethraceae & Clethra scabra Pers. & Carne de vaca \\
\hline Clusiaceae & Kielmeyera grandiflora (Wawra) Saddi & Pau santo \\
\hline Erytroxilaceae & Erythroxylum sp & Fruto de pomba \\
\hline Erytroxilaceae & Erythroxylum suberosum St. Hil. & Galinha choca \\
\hline Euphorbiaceae & Croton floribundus Spreng. & Capixingui \\
\hline Fabaceae & Anadenanthera peregrina (L.) & Angico branco \\
\hline Fabaceae & $\begin{array}{l}\text { Anadenanthera peregrina (L.) Speg. var. falcata } \\
\text { (Benth) }\end{array}$ & $\begin{array}{l}\text { Angico } \\
\text { cascudo }\end{array}$ \\
\hline Fabaceae & Bauhinia rufa (Steud.) & Pata de vaca \\
\hline Fabaceae & Copaifera langsdorffii Desf. & Copaíba \\
\hline Fabaceae & Stryphnodendron pulcherrimum (Willd.) Hochr. & Barbatimão \\
\hline Lauraceae & Nectandra sp & Nectandra \\
\hline Lauraceae & Nectandra cuspidata Nees & Canelão \\
\hline Lauraceae & Ocotea $s p$ & Ocotea \\
\hline Melastomataceae & Tibouchina candolleana (Mart. ex DC.) & Quaresmeira \\
\hline
\end{tabular}




\begin{tabular}{lll}
\hline Melastomataceae & Miconia ligustroides (DC.) Naudin & Jacatirão \\
Myrcinaceae & Rapanea sp & Capororoca \\
Myrtaceae & Myrcia fallax (Rich.) DC. & Guamirim \\
Sapindaceae & Cupania vernalis Cambess. & Camboatá \\
- & Não identificada A & - \\
- & Não identificada B & - \\
- & Não identificada $C$ & - \\
\hline
\end{tabular}

Tabela 2. Estimativa de parâmetros fitossociológicos encontrados por espécie em ordem decrescente do Indice de Valor de Importância (IVI). Legenda: DA: densidade absoluta; DR: densidade relativa (\%); DoR: dominância relativa (\%); FrA: freqüência absoluta; FR: freqüência relativa (\%).

\begin{tabular}{|c|c|c|c|c|c|c|}
\hline Nome científico & DA & DR & DoR & FrA & FR & IVI( $\%)$ \\
\hline Scheflera macrocarpha (Cham. \& Schltdl.) & 0,05 & 0,75 & 27,31 & 2,5 & 0,73 & 28,80 \\
\hline $\begin{array}{l}\text { Anadenanthera peregrina (L.)var. falcata } \\
\text { (Benth) }\end{array}$ & 0,66 & 9,87 & 6,01 & 32 & 9,43 & 25,33 \\
\hline Nectandra cuspidata Nees & 0,76 & 11,37 & 1,92 & 39 & 11,5 & 24,81 \\
\hline Rapanea SP & 0,84 & 12,62 & 0,58 & 37,5 & 11,06 & 24,27 \\
\hline Não identificada $C$ & 0,54 & 8,12 & 1,10 & 36 & 10,61 & 19,85 \\
\hline Copaifera langsdorffii Desf. & 0,60 & 9 & 2,48 & 23,5 & 6,93 & 18,42 \\
\hline Não identificada $B$ & 0,43 & 6,5 & 1,66 & 26 & 7,56 & 15,83 \\
\hline Syagrus romanzoffiana (cham.) glassm & 0,041 & 0,62 & 9,34 & 2,5 & 0,73 & 10,71 \\
\hline Não identificada A & 0,25 & 3,87 & 2,06 & 15,5 & 4,57 & 10,51 \\
\hline Schefflera vinosa (Cham. \& Schltdl.) & 0,34 & 5,12 & 0,45 & 15,5 & 4,57 & 10,15 \\
\hline Cupania vernalis Cambess. & 0,30 & 4,5 & 1,09 & 14 & 4,12 & 9,72 \\
\hline Anadenanthera peregrina (L.) & 0,04 & 0,62 & 8,34 & 2,5 & 0,73 & 9,71 \\
\hline $\begin{array}{l}\text { Stryphnodendron pulcherrimum (Willd.) } \\
\text { Hochr. }\end{array}$ & 0,10 & 1,5 & 6,06 & 6 & 1,76 & 9,33 \\
\hline Gochnatia polympopha Less. & 0,16 & 2,5 & 4,30 & 8,5 & 2,50 & 9,31 \\
\hline Erythroxylum suberosum St. Hil. & 0,11 & 1,75 & 4,66 & 7,5 & 2,21 & 8,62 \\
\hline Myrcia falax (Rich.) DC. & 0,25 & 3,75 & 0,60 & 13 & 3,83 & 8,19 \\
\hline Kielmeyera grandiflora (Wawra) Saddi & 0,10 & 1,62 & 3,60 & 6 & 1,76 & 7,00 \\
\hline Bauhinia rufa (Steud.) & 0,17 & 2,62 & 0,35 & 9,5 & 2,80 & 5,78 \\
\hline Nectanda SP & 0,11 & 1,75 & 1,91 & 5,5 & 1,62 & 5,28 \\
\hline Ilex paraguaiensis A.St.-Hil. & 0,10 & 1,62 & 1,28 & 5,5 & 1,62 & 4,53 \\
\hline Ocotea $S P$ & 0,09 & 1,37 & 1,91 & 4 & 1,17 & 4,47 \\
\hline Xypolia sericea St. Hill. & 0,10 & 1,5 & 1,17 & 5,5 & 1,62 & 4,29 \\
\hline Protium heptaphyllum March. & 0,06 & 1 & 1,67 & 3 & 0,88 & 3,56 \\
\hline Aspidosperma tomentosum Mart. & 0,06 & 1 & 1,23 & 4 & 1,17 & 3,41 \\
\hline Erythroxylum SP & 0,04 & 0,62 & 1,99 & 2,5 & 0,73 & 3,35 \\
\hline $\begin{array}{l}\text { Handroanthus chrysotrichus (Mart. ex DC.) } \\
\text { Mattos }\end{array}$ & 0,05 & 0,75 & 1,83 & 2 & 0,58 & 3,17 \\
\hline Croton floribundus Spreng. & 0,04 & 0,62 & 1,80 & 2,5 & 0,73 & 3,16 \\
\hline Clethra scabra Pers. & 0,06 & 1 & 1,51 & 2 & 0,58 & 3,10 \\
\hline Miconia ligustroides (DC.) Naudin & 0,06 & 1 & 0,87 & 3 & 0,88 & 2,76 \\
\hline \multirow[t]{2}{*}{ Tibouchina candolleana (Mart. ex DC.) } & 0,06 & 1 & 0,65 & 2,5 & 0,73 & 2,39 \\
\hline & 6,69 & 100 & 100 & 339 & 100 & 300 \\
\hline
\end{tabular}


A área em estudo apresentou um valor total de 2,98 para o índice de diversidade de Shannon \& Wiener, valor abaixo do encontrado numa área de Cerrado no sudoeste de Goiás por Rocha et al (2014) que foi de 3,22. Valores encontrados por Almeida et al (2014) em 27 anos de monitoramento de uma área de Cerrado Sensu stricto na Fazenda Água Limpa - DF variaram de 3,27 a 3,49. Ainda que o valor encontrado neste trabalho seja próximo de 3,0, pode-se verificar que ficou abaixo dos encontrados na literatura em várias áreas. Talvez o baixo valor de diversidade deva-se ao estado de conservação da área avaliada, pois é uma área que sofre pressão do entorno além da invasão de espécies exóticas como do gênero Pinus spp.

Em um cerrado sensu stricto em Brasilândia - MG foram encontrados valores de 2,68 a 2,78 em 11 anos de avaliação (SOUZA et al 2011) Felfili et al (1997), estudando 11 áreas de Cerrado sensu stricto da Chapada Pratinha e Chapada dos Veadeiros obtiveram índice de diversidade variando de 3,1 a 3,7. Segundo Brower e Zar (1997) uma comunidade tem alta diversidade se muitas espécies igualmente abundantes estiverem presentes, indicando a existência de uma comunidade complexa, pois quanto maior a variedade de espécies maior a variedade de interações.

De acordo com Saporetti Jr. et al. (2003) valores acima de 3,11 para o índice de Shannon \& Wiener indicam formações vegetais bem conservadas. Desta maneira com um índice de 2,98 podemos afirmar que a invasão de espécies na área estudada da EEI tem sido prejudicial pois a proximidade com a comunidade do entorno (Figura 2) exerce forte efeito antrópico na diversidade de espécies.

\section{CONCLUSÃO}

A família Fabaceae teve o maior número de espécies amostradas; sendo as espécies Scheflera macrocarpha (Cham. \& Schltdl.), Anadenanthera peregrina (L.) var. falcata (Benth) e Nectandra cuspidata Nees com maiores valores de IVI. A área em estudo apresentou um valor total de 2,98 para o índice de diversidade de Shannon \& Wiener, valor abaixo do comumente encontrado para áreas de cerrado.

Pode-se notar a descaracterização da vegetação de cerrado devido a Estação Ecológica de Itapeva situar-se numa zona de transição de Cerrado e Floresta Estacional e pelo fato de estar cercada por reflorestamento de Pinus sp, o que colabora muito para os efeitos antrópicos encontrados no presente estudo tais como o baixo índice de diversidade quando comparado a outras áreas. Desta maneira pode-se considerar estes resultados para o desenvolvimento de planejamentos de manejo para a Unidade de Conservação (UC).

\section{REFERÊNCIAS BIBLIOGRÁFICAS}

ALMEIDA, R. F.; FAGG, C. W.; OLIVEIRA, M. C.; MUNHOZ, C. B. R.; LIMA, A. S.; OLIVEIRA, L. S. B. Mudanças florísticas e estruturais no cerrado sensu stricto ao longo de 27 anos (1985-2012) na Fazenda Água Limpa, Brasília, DF. Rodriguésia, Rio de Janeiro RJ, v. 1, n. 65, p.001-019. 2014.

ALMEIDA, R.S., SOUZA, S.C.P.M., BAITELlO, J.B., PASTORE, J.A., AGUIAR, O.T., KANASHIRO, M.M., MATTOS, I.F.A., LIMA, C.R.; CIELO-FILHO, R. Campo sujo úmido: Fisionomia de cerrado ameaçada pela contaminação biológica de Pinus elliottii Engelm. na Estação Ecológica de Itapeva, Estado de São Paulo. Revista do Instituto Florestal, v. 22, n. 1, p. 71-91, 2010. 
APG. ANGIOSPERM PHYLOGENY GROUP. An update of the Angiosperm Phylogeny Group classification for the orders and families of flowering plants: APG III. Botanical Journal of the Linnean Society, v. 161, p.105-121, 2009.

ASSUNÇÃO, S. L.; FELFILI, J. M. Fitossociologia de um fragmento de cerrado sensu stricto na APA do Paranoá, DF. Brasil. Acta Botanica Brasilica, Belo Horizonte-MG, v. 18, n. 4, p. 903-909, 2004.

BROWER, J. E.; ZAR, J. H. Field and laboratory methods for general ecology. Duduque: WMC Brown, 1997, 194p.

CIELO-FILHO, R.; AGUIAR, O. T.; BAITEllo, J. B.; PASTORE, J. A.; TONIATO, M. T. Z.; SOUZA, S. C. P. M.; LIMA, C. R.; ALMEIDA, R. S.; COSTA, N. O. Aspectos Florísticos da Estação Ecológica de Itapeva, SP: uma Unidade de Conservação no Limite Meridional do Bioma Cerrado. Biota Neotropical, Campinas-SP, v. 12, n. 2, p, 147-166, 2012.

DURIGAN, G.; NISHIKAWA, D. L. L.; ROCHA, E.; SILVEIRA, E. R.; PULITANO, F. M.; REGAlADO, L. B.; CARVALHAES, M. A.; PARANAGUÁ, P. A.; RANIERI, V. E. L. Caracterização de dois estratos da vegetação em uma área de cerrado no município de Brotas, SP, Brasil. Acta Botanica Brasilica, Belo Horizonte-MG, v. 16, n. 3, p. 251-262, 2002.

DURIGAN, G.; SIQUEIRA, M.F.; FRANCO, G.A.D.C.; BRIDGEWATER, S.; RATTER, J.A. The vegetation of priority areas for cerrado conservation in São Paulo State, Brazil. Edinburgh Journal of Botany, v. 60, n. 2, p. 217-241, 2003.

FELFILI, J. M.; SILVA JUNIOR, M. C.; REZENDE, A . V.; NOGUEIRA, P.E.; WALTER, B. M. T., SILVA, M. A . \& ENCINAS, J. I. Comparação Florística e Fitossociológica do Cerrado nas Chapadas Pratinha e dos Veadeiros. 1997, p. 6-11. In: LEITE, L. \& SAITO, C. H.(ed.). Contribuição ao conhecimento Ecológico do cerrado. Brasília-DF: Ed. Universidade de Brasília, 1997.

FIDELIS, A.T.; GODOY, S.A.P. Estrutura de um cerrado stricto sensu na gleba cerrado Pé de Gigante, Santa Rita do Passa Quatro, SP. Acta Botanica Brasilica, Belo Horizonte-MG, v.17, n. 4, p. 532-539, 2003.

FLORA DO BRASIL. Lista de espécies da flora do Brasil. 2014. Disponível em: http://floradobrasil.jbrj.gov.br/jabot/listaBrasil/PrincipalUC/PrincipalUC.do;jsessionid=CDE7 CBDBEE2C9FC71AE2CBD8FFE4DD10. Acesso em: 01 de maio de 2015.

KREBS, C. J. Ecological methodology. 2 ed. Menlo Park: Addison Wesley Longman, 1999. $620 \mathrm{p}$.

MAGURRAN, A, E. Ecological Diversity and Its Measurement . London: Croom Helm. [A general book on ecological diversity. Models (for the distribution of species)], 1988.

MULLER-DOMBOIS, D.; ELLENBERG, H. Aims and methods of vegetation ecology. New York: Wiley, 1974. 546 p.

NOVAIS, P.S., ROSSI, M., MATTOS, I.F.A.; KANASHIRO, M.M. Os solos da Estação Ecológica de Itapeva: caracterização e mapeamento. IF Série Registros, São Paulo-SP, n. 40, p. 217-222, 2009.

PAUlA, A.; MARTINS, F. Q.; BATALHA, M. A. P.L.; RODRIGUES, R.; MANHÃES, M. A. Riqueza, diversidade e composição florística em áreas de cerrado em regeneração e preservado na estação ecológica de Itirapina - SP. Ciência Florestal, Santa Maria-RS, v. 25, n. 1, p. 231-238, jan-mar., 2015 
REYS, P.; CAMARGO, M. G. G. C.; GROMBONE-GUARATINI, M. T. TEIXEIRA, A. P.; ASSIS, M. A.; MORELLATO, L. P. C. Estrutura e composição florística de um Cerrado sensu stricto e sua importância para propostas de restauração ecológica. Hoehnea, São Paulo - SP, v. 3, n. 40, p. 449-464, 2013.

RIBEIRO, J.F.; WALTER, B.M.T. Fitofisionomias do bioma Cerrado. In: SANO, S.M.; ALMEIDA, S.P. (Eds.). Cerrado: ambiente e flora. Planaltina: EMBRAPA, 1998. p. 89-166.

ROCHA, E.; REYS, P.; SILVA, P. O.; SOARES, M. P. Florística e fitossociologia em um fragmento de cerrado no sudoeste de goiás. Global Science and Technology, Rio VerdeGO, v. 07, n. 03, p.110 - 118, set/dez. 2014.

SANQUETTA, C.R; PIZATOO, W.; NETTO, S.P.; FIGUEIREDO FILHO, A.; EISFELD, R.L. Estrutura vertical de um fragmento de floresta ombrófila mista no centro sul do Paraná. Revista Floresta, Curitiba-PR, v. 32, n. 2, p. 267-276, 2002.

SÃO PAULO (Estado). Atlas das unidades de conservação ambiental do Estado de São Paulo, parte II: interior, Secretaria do Meio Ambiente do Estado de São Paulo. São Paulo-SP: Metalivros, 1998. 30 p.

SAPORETTI JUNIOR. A. W.; MEIRA NETO. J. A. A; ALMADO. R P. Fitossociologia de Cerrado sensu stricto no Município de Abaete-MG. Revista Árvore, Viçosa - MG v. 27, n. 3, p.413-419. 2003.

SOARES. M. P; SANTOS. T. M; DOURADO. D. M; SILVA. P. O. DA; SÁ. J. L DE. Análise fitossociológica do componente arbóreo de um remanescente de cerradão em Rio Verde-Goiás. Global Science and Tecnology. Rio Verde-GO, v. 05, n. 03, p. 87-97, 2012.

SOUZA, F, N,; SCOLFORO, J. R.; SANTOS, R. M.; SILVA, C. P. C. Management systems in an area of cerrado sensu strict. Cerne, Lavras-MG, v. 17, n. 1, p. 85-93, jan./mar. 2011

SPIEGEL, M. R. Estatística. São Paulo: McGraw-Hill, 1976. 357 p

TONIATO, M.T.Z., LEITÃO FILHO, H.F.; RODRIGUES, R.R. Fitossociologia de um remanescente de floresta higrófila (mata de brejo) em Campinas, SP. Revista Brasileira de Botânica, São Paulo-SP, v. 21, n.2, p.197-210, 1998.

TORRES, R.B., MATTHES, L.A.F.; RODRIGUES, R.R. Florística e estrutura do componente arbóreo de uma mata de brejo em Campinas, SP. Revista Brasileira de Botânica, São Paulo-SP, v. 17, n. 2, p.189-194, 1994. 